\title{
Fuzzy Logic Based Control Strategies for an Electromagnetic Actuated Sewing Machine Presser Foot
}

\author{
Florian Guhr $^{(1)}$, Luis F. Silva ${ }^{(2)}$, Filomena Soares ${ }^{(3)}$, Helder Carvalho ${ }^{(4)}$ \\ ${ }^{(1)}$ University of Applied Sciences Esslingen, 73730 Esslingen, Germany \\ ${ }^{(2)}$ Dept. of Mech. Eng., School of Engineering University of Minho, 4800-058 Guimara es, Portugal \\ Email: lffsilva@dem.uminho.pt \\ ${ }^{(3)}$ Dept. of Ind. Elec., School of Engineering University of Minho, 4800-058 Guimar es, Portugal \\ Email: fsoares@dei.uminho.pt \\ ${ }^{(4)}$ Dept. of Tex. Eng., School of Engineering University of Minho, 4800-058 Guimara es, Portugal \\ Email: helder@det.uminho.pt
}

\begin{abstract}
Industrial sewing machines have been significantly improved in recent years and especially electronic sewing control is now being introduced. This study concerns the development of a controller for an electromagnetically actuated presser foot. The controller is responsible for controlling the vertical movement of the presser foot. This is an important point since the presser foot tends to bounce at high sewing speeds, resulting in irregular seams. In this study a fuzzy logic controller was used. The reference displacement value is set up by an adaptive method in order to respect changes on the number of plies. The tested fuzzy logic controller allows a better performance of the control especially in relation with different fabrics. During this study another control strategy was considered. This control strategy combines a PI-algorithm with a fuzzy logic controller. Yet, the controller restricts the use of new fabries, which have not been defined in advance. This aspect will be a major point in the future development in order to recognize the fabrics during sewing and to adapt the controller adequately.
\end{abstract}

Keywords: Fuzzy Logic Controller, Electromagnetic actuated Presser Foot, Sewing, PID Controller

\section{Introduction}

Industrial sewing machines have been improved a lot in the recent years. Most manufactures can support a large field of applications, with machines that can perform operations with high quality and at very high speeds. Yet, some problems arise, mainly due to the constant change in materials being sewn.

One important system of a sewing machine is the material feeding system. The objective of this study is the feeding system of an industrial overlock sewing machine, which is depicted in Fig. 1. The system consists of three components: a presser foot, a throat plate and a feed dog. The throat plate is a smooth surface that supports the fabric being sewn with openings for the needle and the feed dog to pass. During this movement the feed dog rises above the throat plate to engage the fabric against the underside of the presser foot before starting the advancing motion. Some of the problems in the sewing process rely in the interaction between the presser foot and the feed dog. The presser foot is responsible for guaranteeing the required pressure to control the fabric feeding and to constrain their movement during needle penetration and withdrawal.

In order to study the feeding process and to control the presser foot, the sewing machine was instrumented with a LVDT (linear variable differential transformer) for measuring the vertical displacement of the presser foot, and a miniature piezoelectric force transducer to measure the (compression) forces exerted on the presser foot bat.

A proportional force solenoid is used to interact with the presser foot, according to the arrangement presented in Fig. 1. Up to now, the available actuators have a quite large response time (about 50 ms step response), allowing only a stitch-by-stitch control of the maximum displacement point. Future actuators with better response times may permit the control of the whole presser-foot trajectory.

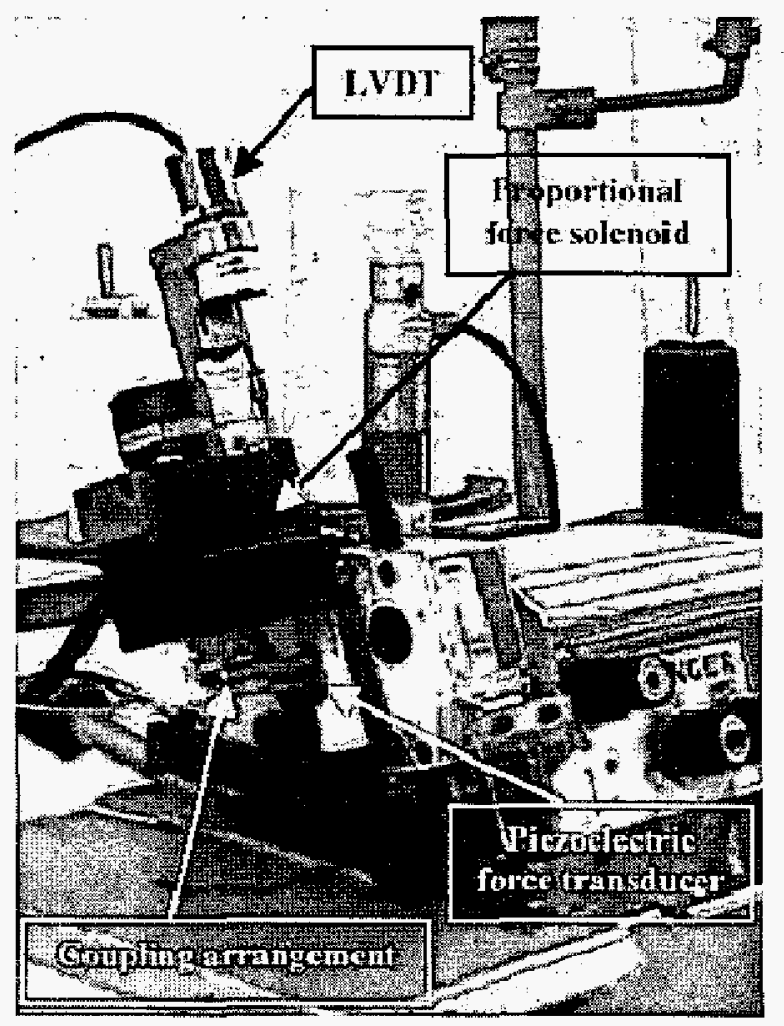

Fig. 1: Industrial overlock sewing machine 
The objective of this controller is to keep the displacement in admissible displacement limits, a strategy more thoroughly explained in [1].

The controllers have been implemented in LabView. The signals from the sensors are conditioned and acquired by an acquisition board. They are then further processed to obtain stitch-by-stitch maximum displacement values. Based on these discrete values the control algorithm computes the output for the actuator.

\section{Adaptive method}

An adaptive method was used for all the presented controllers to set up a new reference value whenever the error or force output exceeds a defined range. The method allows adapting different fabrics and a different number of plies. The problem is that sewing defects like folds, may be incorrectly interpreted and a new reference value may be set, an undesired situation.. Therefore new techniques will be considered in the future to avoid adaptation to these sewing defects.

The definition of references is being implemented by running a specific sewing test before the operation, in order to determine the reference values based on an anti-bouncing condition.

\section{Fuzzy Logic Controller}

Following the research efforts undertaken by the authors in [1] und [2], this section refers to the design of a fuzzy logic controller for controlling the presser foot, presenting and discussing the results obtained during testing.

\subsection{Controller design}

In order to define the controller parameters, some tests were carried out varying the sewing speed and the force output. The displacement of the presser foot was measured during these tests, for a range of sewing speeds and presser-foot forces. In Fig. 2 an example of the characterisation of two plies of an interlock fabric is depicted. In this figure it is possible to observe how the displacement varies with force and speed. In terms of controller design, and based on the result of practical sewing tests, the controller should maintain a displacement range of about $0.05 \mathrm{~mm}$ around a

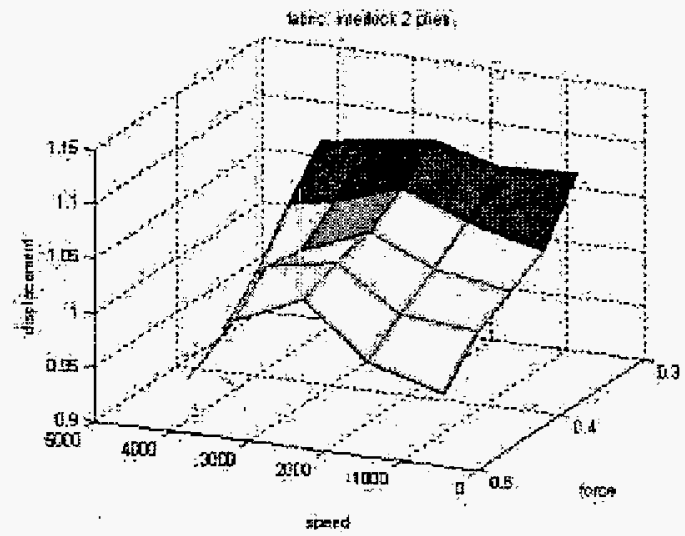

Fig. 2: 3D chart for two plies of an interlock fabric pre-defined average value. The correspondent force range lies between 17.4 to $26.8 \mathrm{~N}$ for a speed variation from 900 to 4700 stitches per minute (spm).

Another interesting fact is that the sewing speed needs also to be taken into account, but seems to be less important.

With the help of these graphs for the defined fabrics, the error range was defined from -0.05 to $0.05 \mathrm{~mm}$ and the force range from $17.4 \mathrm{~N}$ to $26.8 \mathrm{~N}$. This force range seems to manage the defined displacement error over the used speed range. Based on these principles the Fuzzy Logic Controller was designed. Due to the preliminary studies concerning the setting of the displacement reference, it was decided to keep the adaptive method of the reference based on the error range.

The Fuzzy Logic Controller uses three inputs. One of the three inputs is the error based on the reference and the current displacement. In addition to the error, the speed and the current displacement were used as input variables to the Fuzzy Logic Controller to ensure an adaptation on the speed and on the number of plies. The control scheme is depicted in Fig. 3. With these three input variables and the force as output, the member-functions were defined (Fig. 4 to Fig. 7).

In Fig. 4 the speed was defined in a range of 0 to 4700 spm. The member-functions are divided into low ( 0 to $2350 \mathrm{spm}$ ), mid ( 0 to $4700 \mathrm{spm})$ and high speed (2350 to $4700 \mathrm{spm})$. In Fig. 5 the error is depicted as second antecedent. It was fixed from -0.05 to $0.05 \mathrm{~mm}$ and divided into memberfunctions of negative, zero and positive.

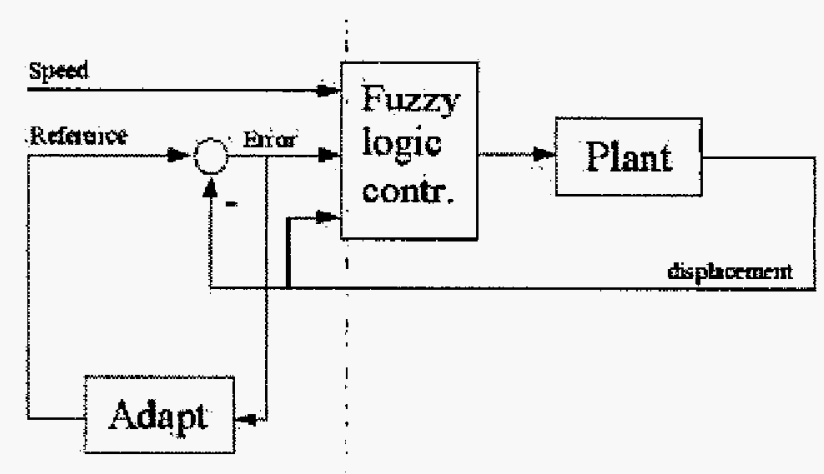

Fig. 3: Fuzzy Logic Controller schema

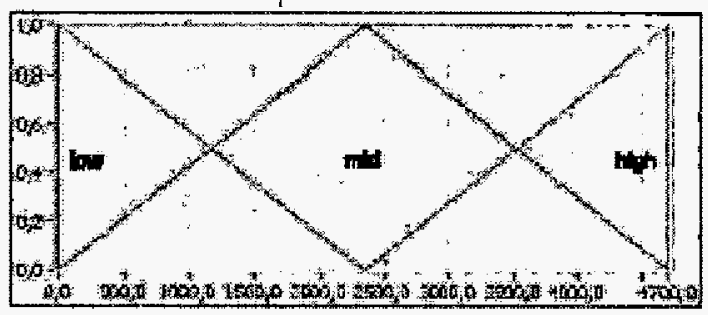

Fig. 4: First antecedence: speed 


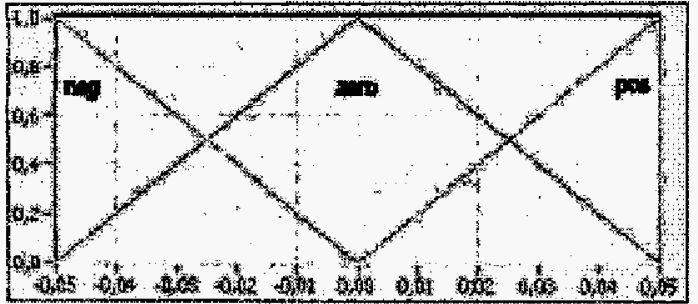

Fig. 5: Second antecedence: error

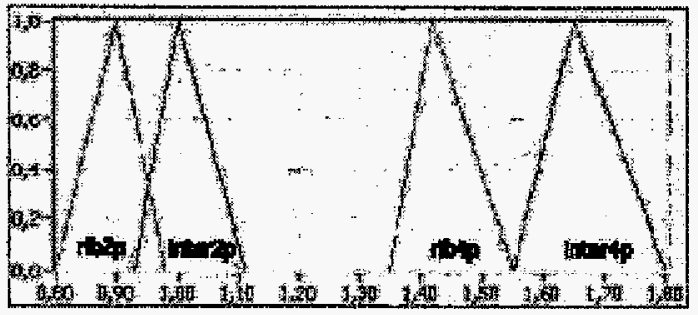

Fig. 6: Third antecedence: displacement

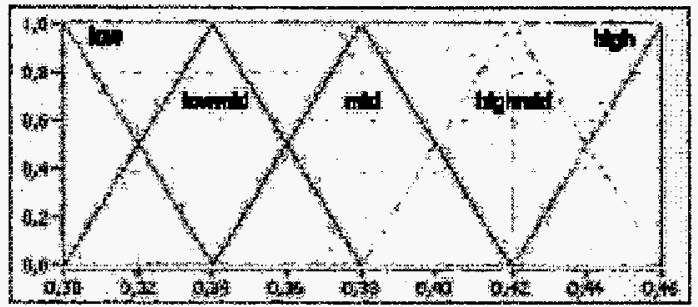

Fig. 7: Consequence: force

As the third antecedent the displacement was used, in order to differentiate between different types of fabrics as well as different number of plies to adapt the force. For this purpose, ranges were defined from 0.8 to $0.98 \mathrm{~mm}$ for two plies of riblx 1 fabric, from 0.93 to $1.12 \mathrm{~mm}$ for two plies of interlock fabric, from 1.35 to $1.55 \mathrm{~mm}$ for four plies of riblx 1 and from 1.55 to $1.80 \mathrm{~mm}$ for four plies of interlock fabric, as the member-functions depicted in Fig. 6. The output force variable was fixed to a range from 17.4 to $26.8 \mathrm{~N}$ based on the experience gained on the sewing process. The member-functions were divided into low (17.4 to $19.8 \mathrm{~N})$, lowmid $(17.4$ to $22.1 \mathrm{~N})$, mid $(19.8$ to $24.4 \mathrm{~N})$, highmid $(22.1$ to $26.8 \mathrm{~N})$ and high $(24.4$ to $26.8 \mathrm{~N})$. These member-functions are presented in Fig. 7.

\subsection{Test results}

The objective is to compare the results of the adaptive Pcontroller (already designed and implemented for this application [3]) with the results of the previously described Fuzzy Logic Controller. A PID-controller did not show a significant improvement related to the used P-controller, therefore it was decided to continue working with a Pcontroller. The comparison between the P-controller and the Fuzzy Logic Controller is based on different types of fabrics and a different number of plies. The P-controller was used with a gain of one. It uses practically the same adaptive reference method based on the force output, because the P-controller uses a gain of one. The parameters of the adaptive mechanisms have been chosen so that the two controllers could be compared. To achieve this, the adaptive range of the Fuzzy Logic Controller was fixed to $0.05 \mathrm{~mm}$ or $2.9 \mathrm{~N}$ for the P-controller. Based on this, the two controllers can be compared without adverse effects. During this test, samples of four plies of riblx 1 fabric and two plies on interlock fabric were used at different speeds. In Fig. 8 to Fig. 11 are depicted the displacement maximum peak (above the throat plate level), the displacement error and the force output of the controller. Fig. 8 shows the output of the Fuzzy Logic Controller for two plies of the interlock fabric and, in Fig. 9, the output of the P-controller. In these figures it can be seen that the error is almost zero for both controllers. To accomplish this objective, the Fuzzy Logic Controller needed to be set up exactly, in order to define the force, which minimizes the error to zero. The displacement measured is depicted as the second part of the graph. The displacement of the presser foot is increasing at the beginning when the fabric is not yet completely fed under the presser foot. The same situation appears at the end, recognizable by the decreasing displacement. The force output is depicted in the third part, being the force of the fuzzy logic controller adjusted to be higher than the one set for the P-controller. The P-controller uses, by default for knitted fabrics, $22.1 \mathrm{~N} 0.38 \mathrm{~V}$, corresponding to a force value suitable for all of the knitted fabrics used in this study. The Fuzzy Logic Controller, on the other hand, offers the possibility to adapt the force offset on the type of fabric, which will be recognized based on the measured displacement.

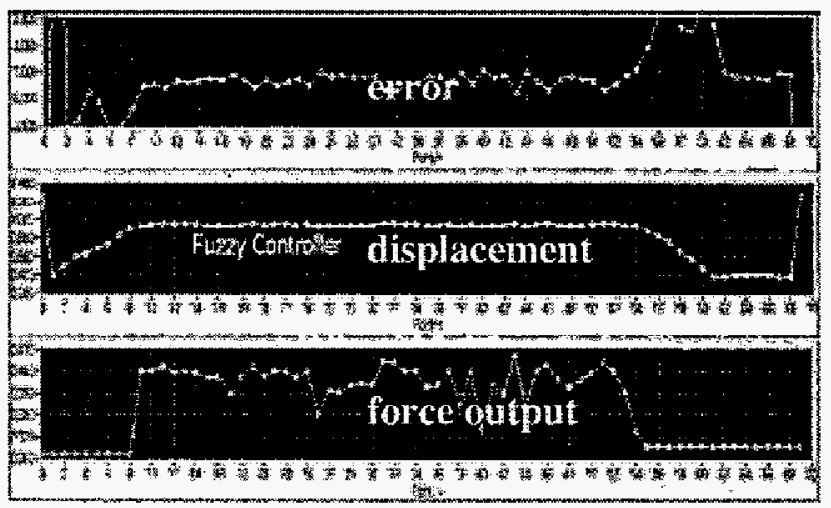

Fig. 8: Error, displacement and Fuzzy Logic Controller output for two plies of an interlock fabric

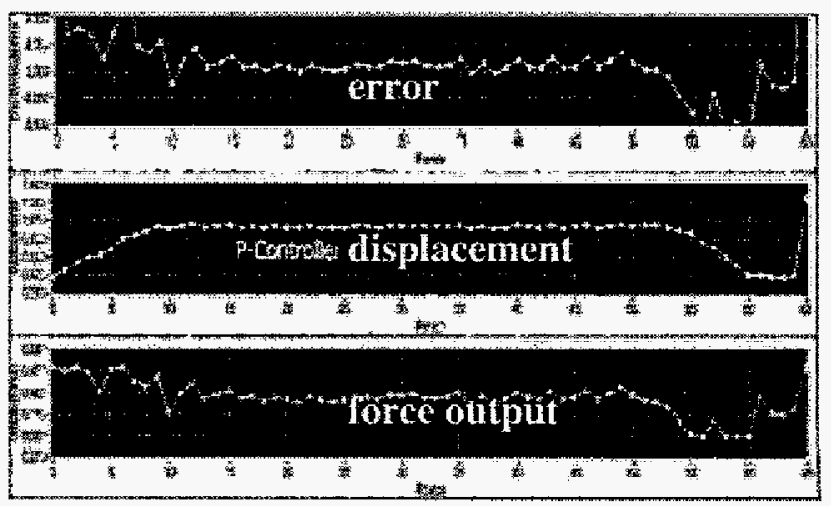

Fig. 9: Error, displacement and P-controller output for two plies of interlock fabric 
In Fig. 10 and Fig 11 are depicted the results for four plies of the riblxI fabric. The output of the Fuzzy Logic controller shows some humps due to the changes of the displacement error. Therefore the Fuzzy Logic Controller needs an improvement by enlarging the error memberfunctions. The P-controller also showed good results, but also revealed a major disadvantage: the P-controller applies the maximum force when the number of plies decreases. This can be seen at the beginning and at the end of the sewing process as presented in Fig 11 .

Therefore the fuzzy logic controller offers more possibilities to define the force output, even for sewing without any fabric. This possibility offers adaptability for different types of fabrics and numbers of plies.

\section{Combined Fuzzy Logic / PID strategy}

This section concerns the combination of the fuzzy logic controller and a PID-controller in a parallel structure. Its design and the obtained results are shown in the following sections.

\subsection{Controller design}

In fact, the function of the combined Fuzzy Logic / PID controller is very similar to the previously described controller. The main difference is that the displacement error will be controlled by the PID-controller whilst the

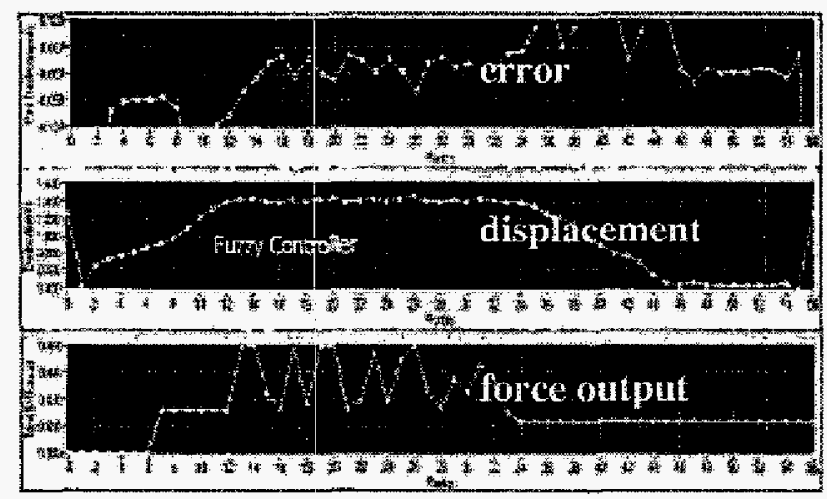

Fig. 10: Error, Displacement and Fuzzy Logic Controller Output for four plies of rib1x1 fabric

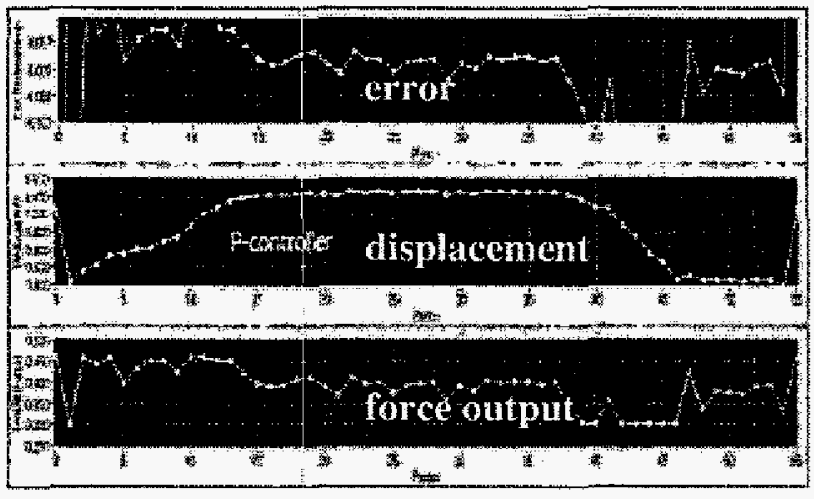

Fig. 11: Error, displacement and P-controller output for four plies of rib1x1 fabric force offset will be adapted by the fuzzy logic controller. Therefore the two controllers are working in parallel to fulfil their different adaptation tasks. In this structure, the fuzzy logic controller serves as an intelligent offset switching control for the force offset, which takes the fabric and the speed into account. In this case we used a PIDcontroller because it seems to be more stable while handling smaller errors, which are due to the combined controller strategy. In figure Fig, 12 is depicted an overview of the controller. While the PID-controller uses the error as input, the fuzzy logic controller uses the speed and the displacement range indicator to determine the force offset. The indicator is determined as the difference between the maximum and minimum displacement, representing the working range of the presser foot, and clearly differentiating different fabrics (with different compressibility) and number of plies. For example, by increasing the number of plies the indicator number is higher than the one obtained using the same number of plies from a different fabric. As already mentioned before, fabric properties like compressibility can also be considered by evaluating the displacement range. Yet, it was just tested as another possibility for the absolute displacement measurement. The force output of this combined controller based on these premises would be something like schematically depicted in Fig. 13. In Fig. 14 to 16 , the fuzzy inference set for the fuzzy logic controller is defined. The used definitions for the speed (Fig. 14) and the force (Fig. 16) are the same as in the previous described controller. Instead of the displacement, the displacement range was used as an indicator for different fabrics or a different number of plies. The definitions for the displacement range are depicted in Fig. 15.

In this figure two plies of interlock (int2p), four plies of interlock (int $4 p$ ), two plies of riblx 1 (rib2p) and four plies of riblx 1 (rib4p) have been defined. In comparison to the definitions of the previous controller, there is now also a definition for no fabric (nofab) - see Fig. 15 -, avoiding severe stress on the mechanical parts and insuring an easier beginning for the feeding process.

The other part of the controller, in this parallel structure, is a PID-controller without offset, because the offset is delivered by the fuzzy logic controller.

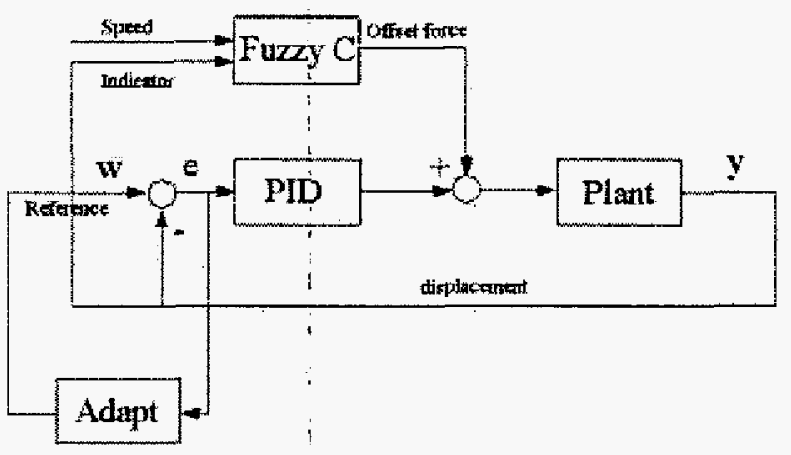

Fig. 12: Controller schema 


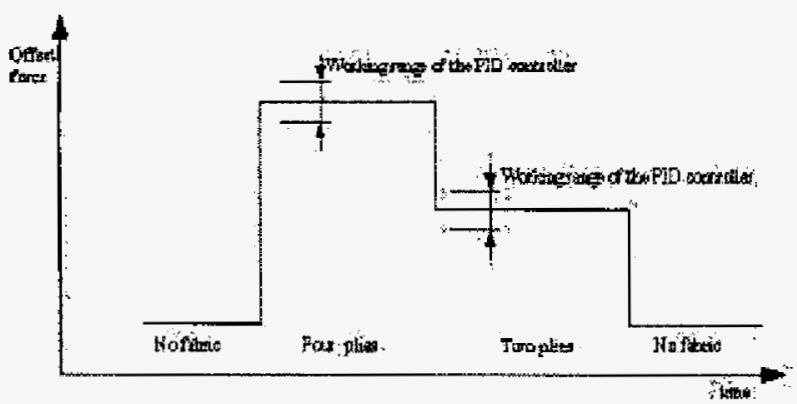

Fig. 13: Main Output

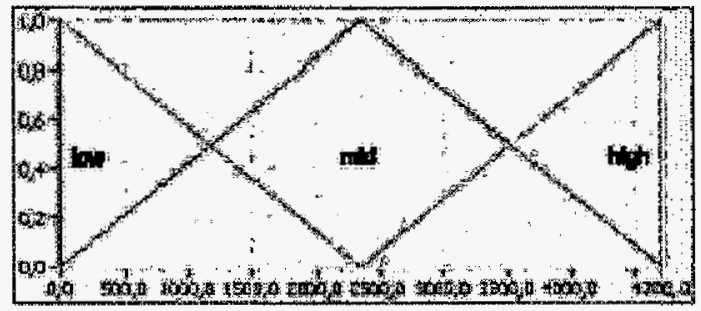

Fig. 14: 1. Antecedence: speed

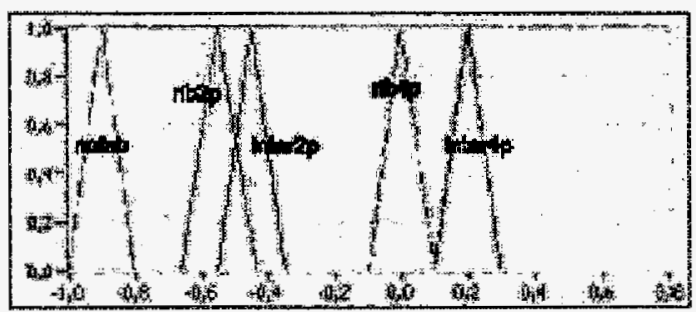

Fig. 15: 2. Antecedence: displacement range

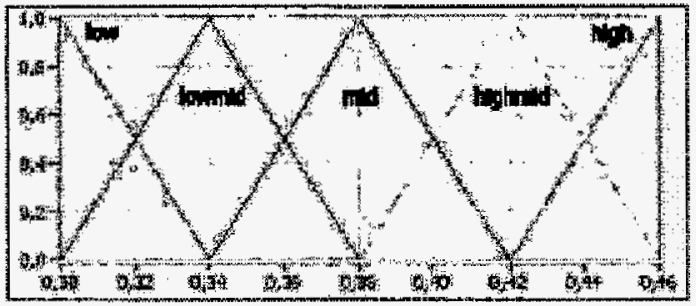

Fig. 16: Consequence: force

Thus the function of the PID-controller is to minimize the error in the defined adaptive range. The adaptive range is fixed by the adaptive mechanism which adapts the reference value. A better possibility, which is being considered for a further development, is the setting of the reference value by another Fuzzy Logic Controller which takes the current displacement as input. This controller will be defined by a preliminary sewing test. To summarize the controller design: the fuzzy logic controller gives a force offset value, which depends on the number of plies, fabric and scwing speed, while the PID is used to vary the force in a smaller range depending on the displacement error.

\subsection{Test results}

The tests were carried out using samples of four changing to two plies. The samples were made of interlock fabric and rib1x1 fabric. The speed of $4000 \mathrm{spm}$ was chosen as the maximum working speed. Fig. 17 represents the measurements using the interlock fabric, depicting the error, the displacement and the controller force output. The displacement clearly indicates the change from four to two plies in the middle of the sample. Fig. 18 depicts the same graphs for the riblx1 fabric. If a closer look is taken to the force output on these two figures, it can be seen that the force offset increases when the number of plies is also increasing. This is due to the definition of the fabrics and the number of plies in the fuzzy logic controller, because more force is needed to press down and to firmly secure a higher number of plies. Therefore, the difference in the force offset can be clearly seen in the force output. In order to respect the type of material and the number of plies, the offset Force was defined depending on the sewing conditions. The force offset for four interlock plies is about $24.4 \mathrm{~N}$ whilst the one for four plies of rib $1 \mathrm{x} 1$ is about $26.8 \mathrm{~N}$. Taking into consideration the second part of the displacement, it can be recognized that the force offset for both fabrics was defined as $22.1 \mathrm{~N}$ (corresponds to $0.38 \mathrm{~V}$ ).

The PID-controller is minimizing the error during sewing. It is responsible for the small changes of the force around the offset. If the error is higher then the predefined range, the new reference value will be set by the adaptive mechanism. The behaviour of the combined controller is better than just the Fuzzy Logic Controller, because the PID-control absorbs the smaller differences.

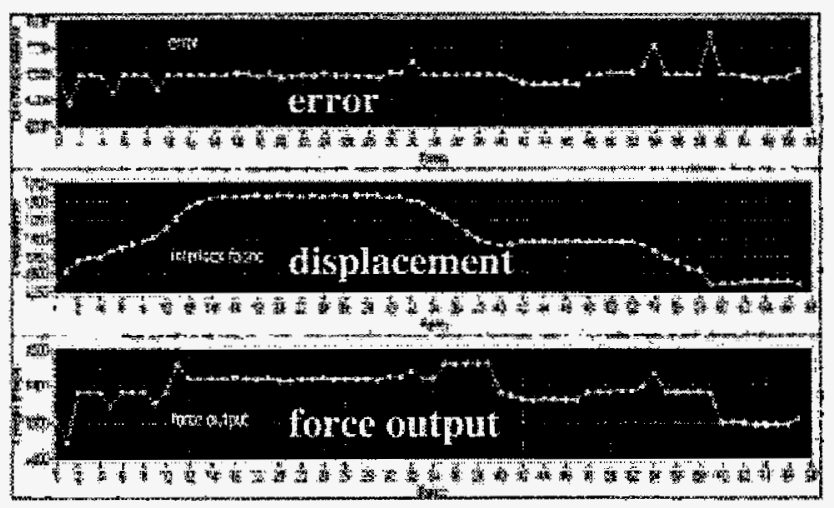

Fig. 17: Еггог, displacement and output of the combined controller using interlock fabric

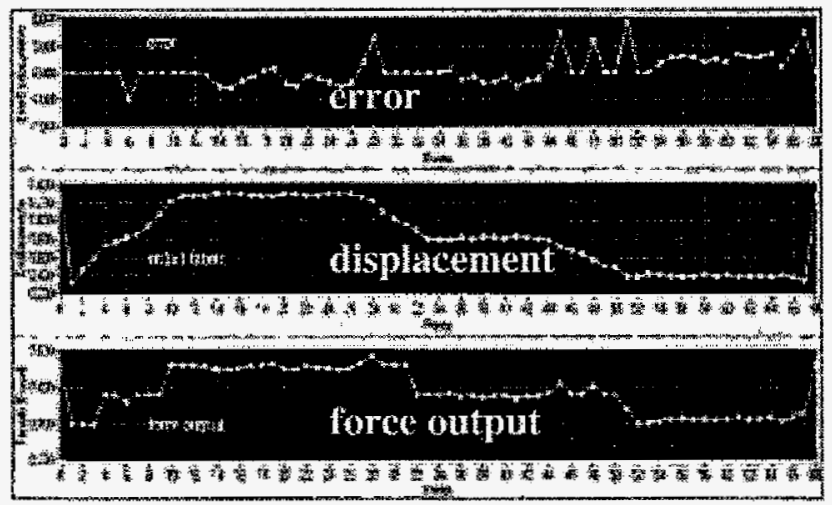

Fig. 18: Error, displacement and output of the combined controller using rib1x1 fabric 
Control based solely on the Fuzzy Logic controller depends entirely and rigidly on a correct tuning of the controller to the fabrics, and thus on a correct "teach-in" process.

\section{Conclusions}

The objective was the development of fuzzy logic based controller strategies. During this work, a Fuzzy Logic Controller was developed and tested. Besides the Fuzzy Logic Controller, another control strategy was implemented based on a parallel structure combing the Fuzzy Logic Controller with a PID-structure as an auxiliary method to correct only small errors.

In order to evaluate the Fuzzy Logic Controller and the combined controller, a comparison with a previously developed reference adapting $P$-controller was made. This simple P-controller just uses the error as feedback variable, with a constant force offset ${ }^{l}$.

The comparison of the adapting P-controller and the adapting Fuzzy Logic Controller revealed that the Fuzzy Controller offers more possibilities of specific adaptation of the sewing parameters like speed, number of plies and type of fabric. Nevertheless, this controller requires more testing and verification while establishing the rules for a desirable operation a fine-tuning of the force, depending on the error, demands a close verification. The Fuzzy Logic Controller can be improved by additional member-functions for the error, which will define its behaviour if the error exceeds the defined range. This behaviour will cover the transition zones while changing the number of plies. This is, for example, one advantage compared to the $\mathbf{P}$. controller, which just gives maximum or minimum force output at this transition phase.

An adaptation to sewing speed was implemented as a property of the fuzzy controller. The P-controller is indirectly influenced by sewing speed: if a higher sewing speed produces larger errors, the p-control will react to them and thus be indirectly influenced by speed. That's why the results of the P-controller and Fuzzy Logic Controller are similar for the tested sewing speeds.

The used Fuzzy Logic controller adapts also to the number of plies and the type of fabric based on the displacement. This feature was not possible with a P- or PID-controller. Therefore an adaptation of the force ranges is needed for the P-controller.

The disadvantage of not adapting the number of plies or type of fabric or speed, led to the development of a parallel controller structure. This paraliel controller structure was made of a Fuzzy Logic Controller and a PID-Controller.

The task of the Fuzzy Logic Controller was set-up a force offset depending on the sewing parameters. The PIDController served to eliminate the error between the reference value and the actual displacement measured.

The tested controller structure seems to be easier to handle, because it does not need a careful calibration like the Fuzzy Logic Controller tested before. The recognition of the

\footnotetext{
${ }^{1}$ It had been previously observed that a simple P-control produced better results than a PID-control.
}

fabrics and the number of plies depends on the definitions of the member functions in the Fuzzy Logic Controller. This definition is fixed in the controller and therefore not applicable for new types of fabrics.

\section{Future Prospects}

Future work will be directed to the development of a method for producing a preliminary "teach-in" sewing test. This test will be used to characterize the fabric in terms of speed, necessary force and optimal reference value, since these are some of the major process parameters in sewing. This can be achieved, for example, by running a test cycle with force variation to determine the displacement range of the fabric. To adapt the necessary force for a given fabric, it could be suitable to have a routine to monitor the "bouncing" of the presser foot. Based on this information, the force offset and reference displacement can be extracted. If the test is run for a different number of plies, the basic values can be defined in the Fuzzy Logic Controller's characteristic. This will serve later to distinguish the different numbers of plies and to set up a proper offset force and reference value. The PID-controller can then be used in parallel to the Fuzzy Logic Controller to correct normal variations or small inaccuracies in the control characteristic of the main controller.

The preliminary definitions created for a fabric can be stored in a database for future use. For each fabric, these parameters should cover speed, force and definitions for a higher number of plies. They can be obtained in offline process planning instruments and downloaded to the machines when necessary. The teach-in methods should be automatic and simple to carry out.

\section{References}

[1] L. F. Silva, M. Lima, and H. Carvalho, et al, "Actuation, monitoring and closed loop-control of sewing machine presser foot", The Institute of Measurement and Control, vol. 25, pp. 419-432, 2003.

[2] L. F. Silva, H. Carvalho, and F. Soares, "Improving feeding efficiency of a sewing machine by on-line control of the presser foot", Proceedings of the International Conference on Ad anced Engineering Design - AED' 2004 (CD-ROM), University of Glasgow, Glasgow, UK, 5-8 September, 2004.

[3] L. F. Silva, F. Soares, and H. Carvalho, "PDD closed-loop adaptive control of an electromagnetic actuated presser foot for overlook sewing machines", to be accepted for the International Conference on Mechatronics - ICOM' 2005. 